\title{
Intense Terahertz Excitation of Semiconductors
}

\author{
S. D. Ganichev and W. Prettl \\ University of Regensburg \\ Germany
}




\section{CONTENTS}

Introduction 1

1 Experimental technique 5

1.1 Sources of high-power terahertz radiation 5

$\begin{array}{lll}1.1 .1 & \mathrm{CO}_{2} \text { laser } & 7\end{array}$

1.1.2 Optically pumped terahertz molecular lasers $\quad 11$

$\begin{array}{cc}\text { 1.1.3 Tunable high-pressure } \mathrm{TE}-\mathrm{CO}_{2} \text { laser pumped } & \\ \text { terahertz molecular lasers } & 16\end{array}$

$\begin{array}{ll}\text { 1.1.4 Free-electron lasers } & 21\end{array}$

$\begin{array}{ll}\text { 1.1.5 Short coherent terahertz pulses } & 30\end{array}$

1.2 Optical components and methods in the $\mathrm{THz}$ range 34

$\begin{array}{lll}\text { 1.2.1 Windows } & 34\end{array}$

$\begin{array}{lll}1.2 .2 & \text { Filters } & 40\end{array}$

$\begin{array}{lll}1.2 .3 & \text { Polarizers } & 55\end{array}$

$\begin{array}{ll}1.2 .4 & \text { Spectral analysis }\end{array}$

$\begin{array}{lll}\text { 1.2.5 Attenuators } & 62\end{array}$

1.2.6 Transmission through the atmosphere, liquid
nitrogen, and liquid helium

1.3 Detection of pulsed terahertz radiation 64

$\begin{array}{lll}\text { 1.3.1 Photon drag detectors } & 67\end{array}$

$\begin{array}{ll}\text { 1.3.2 } \mu \text {-photoconductivity detectors } & 69\end{array}$

$\begin{array}{ll}\text { 1.3.3 Pyroelectric detectors } & 70\end{array}$

$\begin{array}{ll}\text { 1.3.4 Atomic layer thermopile } & 72\end{array}$

1.3.5 New detection concepts $\quad 75$

$\begin{array}{ll}\text { References } & 79\end{array}$

$2 \quad$ Tunneling in terahertz fields 93

2.1 Terahertz field induced tunneling $\quad 94$

2.1.1 Ionization of deep impurities $\quad 94$

2.1.2 Theory of tunneling ionization 98

2.1.3 Phonon-assisted tunneling ionization in the 116

2.1.4 Tunneling ionization in the high-frequency limit 118

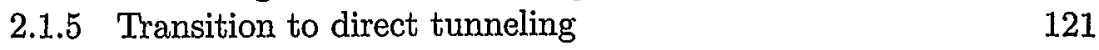

2.1.6 Magnetic field effect on tunneling ionization 124

$\begin{array}{cc}\text { 2.1.7 Tunneling ionization of charged impurities and } & \\ \text { the Poole-Frenkel effect } & 125\end{array}$

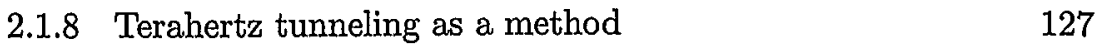

2.2 Photon mediated tunneling in quantum wells 128 
2.2.1 Resonant tunneling

2.2.2 Negative differential conductivity 133

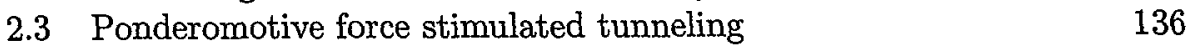

2.3.1 Radiation pressure enhanced tunneling 138

2.3.2 Tunneling enhancement in the near-zone field 145

References

3 Multiphoton excitation 161

$\begin{array}{lll}3.1 & \text { Two-photon absorption } & 162\end{array}$

3.2 Fully developed multiphoton transitions 165

3.2.1 Nonlinear transmission 167

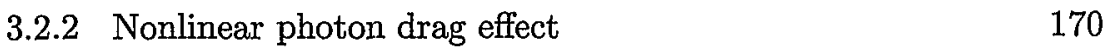

$\begin{array}{lll}\text { 3.3 Multiphoton transitions in low-dimensional systems } & 175\end{array}$

$\begin{array}{ll}\text { References } & 181\end{array}$

4 Saturation of absorption $\quad 185$

$\begin{array}{lll}4.1 & \text { Basics of optical saturation } & 187\end{array}$

$\begin{array}{lll}\text { 4.1.1 Rabi oscillations } & 187\end{array}$

4.1.2 Relaxation $\quad 189$

$\begin{array}{lll}4.2 & \text { Low-power saturation } & 195\end{array}$

4.2.1 Nonlinear spectroscopy of impurities $\quad 195$

4.2.2 Nonlinear cyclotron resonance 201

4.3 Saturation of inter-subband transitions in wide QWs 204

4.4 Reduction of depolarization shift in QWs 206

4.5 Saturation of direct transitions in bulk $p-\mathrm{Ge} \quad 209$

4.6 Pump-and-probe technique 213

4.7 Spin-sensitive bleaching 214

$\begin{array}{ll}4.8 \text { Rabi oscillations } & 219\end{array}$

$\begin{array}{ll}\text { References } & 224\end{array}$

$5 \quad$ Electron gas heating $\quad 237$

5.1 Electron temperature limit and $\mu$-photoconductivity 238

5.2 Heating and absolute electron gas cooling 239

$\begin{array}{lll}5.3 & \text { Dynamic inversion of } \mu \text {-photoconductivity } & 240\end{array}$

5.4 Nonlinear $\mu$-photoconductivity in $2 \mathrm{DEG} \quad 243$

5.4.1 Si-MOSFET structures 244

5.4.2 III-V-compound low-dimensional structures 247

$\begin{array}{lll}5.5 & \text { Light impact ionization } & 249\end{array}$

5.5.1 Bulk InSb 251

5.5.2 Quantum well structures 255

$\begin{array}{ll}5.6 \text { Heating of phonons } & 256\end{array}$

$\begin{array}{ll}\text { References } & 260\end{array}$

6 Terahertz nonlinear optics 269

6.1 Nonlinear optical susceptibility 270

6.2 Terahertz second-order nonlinearity 273 
6.3 Third-harmonic generation and four-wave mixing 278

$\begin{array}{ll}6.4 \text { Terahertz electrooptics } & 280\end{array}$

6.4.1 Terahertz side-bands of near-infrared radiation 280

6.4.2 Dynamic Franz-Keldysh and Stark effects 282

6.5 THz nonlinearities in semiconductor superlattices 284

$\begin{array}{ll}\text { References } & 285\end{array}$

7 Terahertz radiation induced currents 291

$\begin{array}{lll}7.1 & \text { Photon drag effect } & 293\end{array}$

$\begin{array}{ll}\text { 7.1.1 Direct optical transitions } & 294\end{array}$

$\begin{array}{lll}\text { 7.1.2 Drude absorption } & 306\end{array}$

$\begin{array}{cc}\text { 7.1.3 Photoionization of impurities and transitions } & \\ \text { between Zeeman-split levels } & 308\end{array}$

$\begin{array}{lll}7.2 & \text { Linear photogalvanic effect } & 311\end{array}$

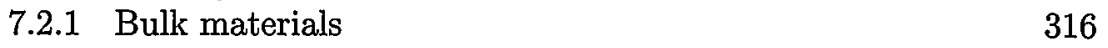

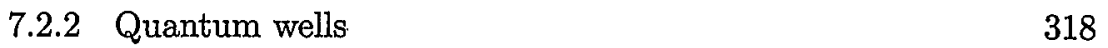

7.2.3 Magneto-photogalvanic effects $\quad 322$

7.2.4 Magneto-gyrotropic effects in quantum wells 325

$\begin{array}{lll}7.3 & \text { Spin photocurrents } & 331\end{array}$

7.3.1 Circular photogalvanic effect 332

$\begin{array}{lll}\text { 7.3.2 Spin-galvanic effect } & 349\end{array}$

7.3.3 Circular photogalvanic effect versus

$\begin{array}{ll}\text { 7.3.4 Application of spin photocurrents } & 361\end{array}$

7.3.5 Monopolar spin orientation 364

$\begin{array}{ll}\text { References } & 367\end{array}$

8 Bloch oscillations $\quad 379$

8.1 Superlattice transport $\quad 380$

8.1.1 Miniband conductance 381

8.1.2 Wannier-Stark hopping 383

8.1.3 Sequential tunneling 384

8.1.4 Interplay between transport mechanisms 385

8.2 $\mathrm{THz}$ excitation of superlattices 386

8.3 Dynamic localization and negative conductivity 391

$\begin{array}{ll}\text { 8.4 } \mathrm{THz} \text { gain in superlattices } & 393\end{array}$

$\begin{array}{ll}\text { References } & 395\end{array}$

A Removal of spin degeneracy $\quad 399$

A.1 Zinc-blende structure based QWs 401

$\begin{array}{ll}\text { References } & 403\end{array}$

B Symbol Glossary-Index $\quad 407$

Index $\quad 413$

Acknowledgement for reuse of figures $\quad 417$ 